\title{
A Universally Defined Standard to Evaluate the Mechanical Stability of Microtextured Surfaces is Necessitated
}

ISSN: 2576-8840

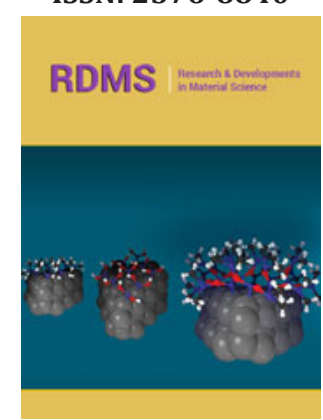

*Corresponding author: Huichao Jin, Key Laboratory of Bionic Engineering, Ministry of Education, Jilin University, Changchun 130022, China

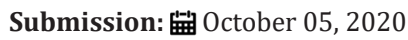

Published: 制October 15, 2020

Volume 14 - Issue 2

How to cite this article: Huichao Jin. A Universally Defined Standard to Evaluate the Mechanical Stability of Microtextured Surfaces is Necessitated. Res Dev Material Sci. 14(2). RDMS.000832. 2020. DOI: 10.31031/RDMS.2020.14.000832

Copyright@ Huichao Jin. This article is distributed under the terms of the Creative Commons Attribution 4.0 International License, which permits unrestricted use and redistribution provided that the original author and source are credited.

\section{Huichao Jin*}

Key Laboratory of Bionic Engineering, Ministry of Education, Jilin University, China

\section{Opinion}

The poor mechanical stability of microtextured surfaces is a major barrier to their engineering applications [1,2]. Accordingly, researchers have devoted themselves to developing microtextured surfaces with robust mechanical stability. Recently, Wu et al. [3] reported a superhydrophobic coating with a robust hierarchical structure and compared its mechanical stability to that of the other robust surfaces. The authors claimed that the test conditions used to evaluate the mechanical properties were the most rigorous as compared to those used in previously reported tests. As there is no universal standard to quantify the mechanical stability of a surface, the following questions are worth discussing: "how was the comparison carried out?" and "what test conditions qualify as rigorous?"

Currently, several methods have been widely used to evaluate the mechanical stability of microtextured surfaces, including sandpaper abrasion, blade scraping, finger wiping, tape peeling, and sand impact. After these tests are performed, researchers usually use the changes in the Water Contact Angle (WCA) to evaluate the mechanical stability of microtextured surfaces [4]. However, the specific testing methods are many and varied in the literature [3]. For example, the sandpaper abrasion test involves several parameters, such as sandpaper grit, load pressure, movement speed, and cycles or distances. Different researchers tend to use different parameters to conduct abrasion tests [5-10]. The selection of a particular parameter may render the experiment easier to conduct or lead to a better result. These practices make it difficult to compare the mechanical property of a microtextured surface with other robust surfaces.

To overcome the chaotic status quo, researchers need to develop a standard to evaluate the mechanical stability of microtextured surfaces. Again, we consider the case of sandpaper abrasion. The combination of sandpaper grit, load pressure, movement speed, cycles, or distances should have a universal standard. Table 1 shows an assumed and fancied standard, in which sandpaper abrasion tests are classified into two categories, namely: (i) low grit test and (ii) high grit test. The combination of both grits is expected to better demonstrate the mechanical property of a microtextured surface. After the abrasion tests, the changes in the WCA of the microtextured surfaces can be measured. Subsequently, the descent rate of the WCA can be calculated with the following equation:

$$
\text { Descent rate } \%=\frac{W C A_{\text {pristine }}-W C A_{\text {abrasion }}}{W C A_{\text {pristine }}} \times 100
$$

Table 1: An assumed sandpaper abrasion standard.

\begin{tabular}{|c|c|c|c|c|}
\hline Test type & Sandpaper grade (Grit) & Load (g) & Speed (m/s) & Cycles \\
\hline Low grit & 100 & 250 & 0.05 & 50 \\
\hline High grit & 1000 & 250 & 0.05 & 50 \\
\hline
\end{tabular}


The microtextured surface with a lower descent rate indicates a higher mechanical stability. In the low- and high-grit abrasion tests, we can obtain two descent rates, which can be used to carry out an effective comparison. Apart from sandpaper abrasion, other test methods (e.g., blade scraping, finger wiping, tape peeling, and sand impact) also need a standard for measurement. If researchers adopt a universal standard, the comparison of the mechanical stability of various microtextured surfaces will be easily achieved.

The purpose of this paper is not to question or criticize the work of $\mathrm{Wu}$ et al. [3] because the issue of effectively comparing the mechanical stabilities of different microtextured surfaces is a problem encountered by many researchers. We hope that the above discussion will inspire researchers to create a standard to evaluate the mechanical stability of microtextured surfaces, which will be beneficial to the development of microtextured surfaces.

\section{References}

1. Wang D, Sun Q Hokkanen MJ, Zhang C, Lin FY, et al. (2020) Design of robust superhydrophobic surfaces. Nature 582(7810): 55-59.

2. Pan S, Chen M, Wu L (2020) Smart superhydrophobic surface with restorable microstructure and self-healable surface chemistry. ACS Applied Materials \& Interfaces 12(4): 5157-5165.

3. Wu B, Lyu J, Peng C, Jiang D, Yang J, et al. (2020) Inverse infusion processed hierarchical structure towards superhydrophobic coatings with ultrahigh mechanical robustness. Chemical Engineering Journal 387: 124066 .

4. Zhu T, Cheng Y, Huang J, Xiong J, Ge M, et al. (2020) A transparent superhydrophobic coating with mechanochemical robustness for antiicing, photocatalysis and self-cleaning. Chemical Engineering Journal 399: 125746.

5. Zhang D, Li L, Wu Y, Zhu B, Song H (2019) One-step method for fabrication of bioinspired hierarchical superhydrophobic surface with robust stability. Applied Surface Science 473: 493-499.

6. Liu S, Wan W, Zhang X, De Crema A, Seeger S (2020) All-organic fluorinefree superhydrophobic bulk material with mechanochemical robustness and photocatalytic functionality. Chemical Engineering Journal 385: 123969.

7. Long C, Qing Y, An K, Liu C, Chai M, et al. (2020) Super wettable bulk Janus materials with mechanical robustness and underwater selfcleaning action. Chemical Engineering Journal 385: 123920.

8. Wang F, Pi J, Song F, Feng R, Xu C, et al. (2020) A superhydrophobic coating to create multi-functional materials with mechanical/chemical/ physical robustness. Chemical Engineering Journal 381: 122539.

9. Celik N, Torun I, Ruzi M, Esidir A, Onses MS (2020) Fabrication of robust superhydrophobic surfaces by one-step spray coating: Evaporation driven self-assembly of wax and nanoparticles into hierarchical structures. Chemical Engineering Journal 396: 125230.

10. Ahuja P, Ujjain SK, Urita K, Furuse A, Moriguchi I, et al. (2020) Chemically and mechanically robust SWCNT based strain sensor with monotonous piezoresistive response for infrastructure monitoring. Chemical Engineering Journal 388: 124174.

For possible submissions Click below: 